\title{
Numerical and Experimental Analysis of Mechanical Loads on Stent- Vessel-Systems
}

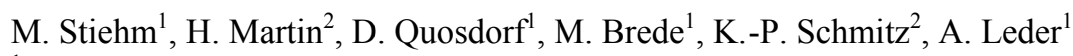 \\ ${ }^{1}$ Chair of Fluid Mechanics, University of Rostock, Rostock, Germany, michael.stiehm2@uni-rostock.de \\ ${ }^{2}$ Institute for Biomedical Engineering, University of Rostock, Rostock, Germany
}

\begin{abstract}
Blood vessels as well as the blood flow are significantly influenced by coronary stents. On the one hand the compliance of the vessel wall will increase due to the stiffness of the stent struts. Therefore a structural mechanic analysis is necessary to find a compromise between the elasticity of the stent and the stiffness necessary to brace the vessel wall.

On the other hand, the stent struts induce non-physiological flow effects like recirculation zones, which result in alterations of the wall shear stress distribution. Particularly low wall shear stresses are under suspect to increase the risk of an in-stent restenosis. In this paper structural und fluid mechanical methods are presented to analyse the influence of the stent on the wall of the vessel and on the blood flow. It has been proven that coronary stent increases the compliance of the vessel wall. Furthermore recirculation zones have been found upstream and downstream of the stent struts. The acquired results will be used to improve the stent design.
\end{abstract}

\section{Introduction}

Coronary stents have been shown to interfere with the blood flow in the stented blood vessel. Therefore in future stent design the stent layout has to be adapted with respect to both, structural and fluid mechanics. The aim of stent development is an implant which does not compromise the mechanics of the vessel and which preferably contributes to healing.

The main task of the stent is to prevent the collapse of the dilated stenosis. Therefore, the stented region has to be buttressed but it should be stiffened as little as possible. One concept to avoid permanent stiffening is the biodegradable stent. Another possible solution is to use selfexpanding stents with hyperelastic material properties. However, all stents at least cause a temporary mismatch of structural vessel properties which has to be investigated.

A further aspect of stent implantation is the alteration of the flow field. The protrusion of the stent struts causes non-physiological flow effects like recirculation and vorticity which lead to a change of the wall shear stress distribution [1]. Several clinical studies have pointed out that especially low wall shear stress correlates with the in-stent restenosis [7, 8, 9]. The optimization of the stent design regarding to fluid mechanical properties seems to be one of the key elements to improve post-implantational complications. Berry et al. for example mentioned that huge spacing between stent struts have a positive influence on the risk for a restenosis.

The Micro-Particle-Image velocimetry has been established for the experimental fluid mechanical investigations, whereas CFD was used for the numerical flow analysis.

The numerical method was validated with the data acquired experimentally. Therefore, for the numerical simu- lations the experimental setup regarding to rheological fluid properties, geometry and boundary conditions are modelled.

\section{$2 \quad$ Material and Methods}

\subsection{Structural Mechanics}

Finite element models of stents with a vessel model with stenosis are investigated using the finite element software ABAQUS. The models include the balloon, the stent and the stenosis model with the following vessel components (stent material, adventitia, media, intima, calcified and lipide plaque). The material properties of these components are taken from the literature [11]. A typical model of a bioabsorbable polymer stent with stenosis is shown in Figure 1.

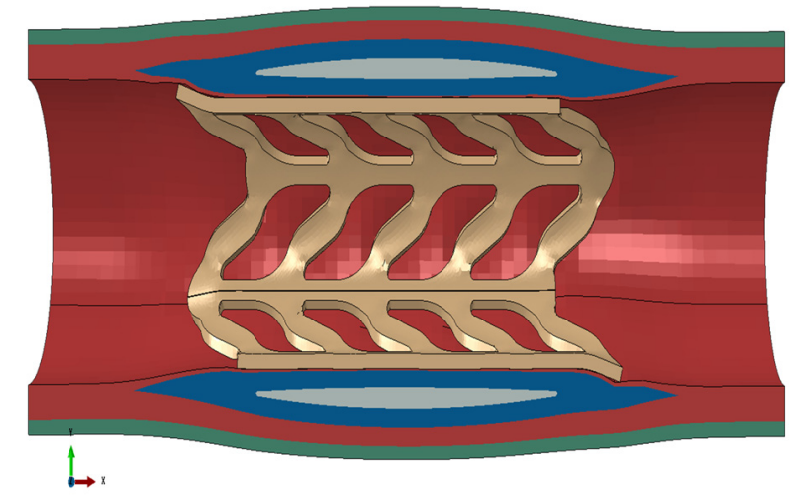

Figure 1 Finite element model of a polymer stent with stenosis showing the different materials

The double contact between stent/balloon and stent/stenosis is considered. The following load cases were analyzed statically:

- Stent dilatation and recoil 
- Establishment of the contact of the stent with the stenosis

- $\quad$ upper and lower physiological pressure.

The compliance and the vessel stress is calculated and compared for the several stent designs.

\subsection{Experimental flow studies}

For the experimental flow studies a generic model stent is used, see Figure 2. The model stent resembles the design features from real stent models. Three model stents were investigated with different cross sections $(\mathrm{B}=0.4 \mathrm{~mm}, \mathrm{~L}=$ $1.0 \mathrm{~mm}, \mathrm{~T}=0.06 \mathrm{~mm}, 0.1 \mathrm{~mm}$ or $0.14 \mathrm{~mm}$ ).

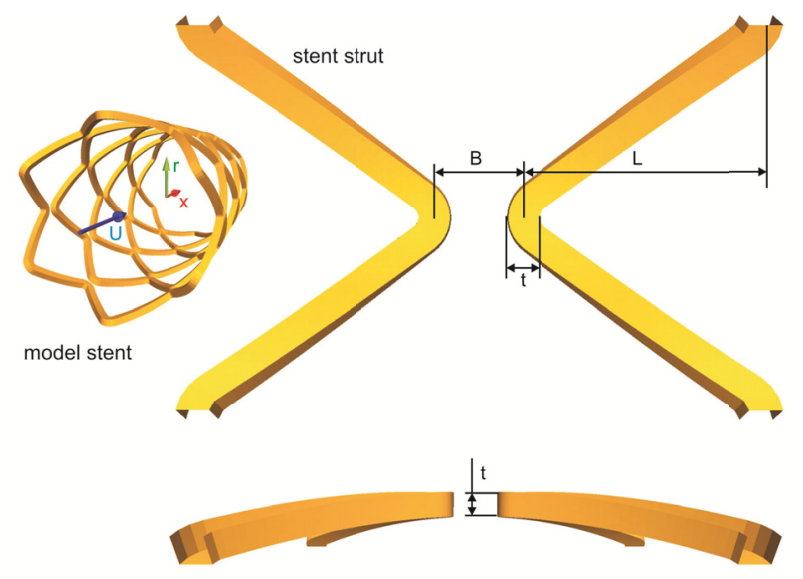

Figure 2: Model stent geometry

The experimental flow studies are performed using a Micro-Particle-Image-Velocimetry setup. The main components are a micro flow channel, a microscope Leica DM $2500 \mathrm{MH}$ equipped with an objective lens (magnification $\mathrm{M}=10 \mathrm{x}$ ) and a CCD-camera. The flow field was illuminated by a Nd:YAG double pulse laser whose beam was coupled into the optical path of the microscope via a liquid light guide.

The flow channel consists of two flow cycles. A nonNewtonian, transparent blood analogue fluid (water, glycerin $23.5 \%$, xanthan $0.02 \%$, potassium thiocyanate $22 \%$ $23 \%$ ) is convected in the main cycle trough an inner channel with a silicone model of the stented vessel $[3,4,10]$. The shear thinning behavior was matched to that of human blood using data obtained by Chien [5]. A second flow cycle contains a $57 \%$ mixture of glycerin and water which is lead through the rectangular outer channel as a bypass to the model. Both fluids are matched to the index of refraction of the silicone model to avoid optical distortions or shadowing. Since the index of refraction depends on the temperature, both fluids are heated up to a temperature of $\mathrm{T}=30^{\circ} \mathrm{C}$ with the help of a heating thermostat.

A set of image pairs is acquired, then the velocities are obtained by a particle tracking algorithm [6] which calculates the particle trajectories of each image pair.

An averaging procedure is used to sample particle trajectories over small interrogation areas. Then a mean velocity for each area was calculated. The resulting velocity field can be used to calculate the velocity gradients. By considering the gradient $\dot{\gamma}=d u / d r$ as the main contributor and taking into account the non-Newtonian viscosity of the fluid $\eta(\dot{\gamma})$ the wall shear stress can be obtained by $\tau=\eta(\dot{\gamma}) \cdot \dot{\gamma}$. The near wall velocity gradient $d u / d r$ is determined by using a linear curve fit and an extrapolation to the vessel wall. Figure 3 displays the experimental setup.

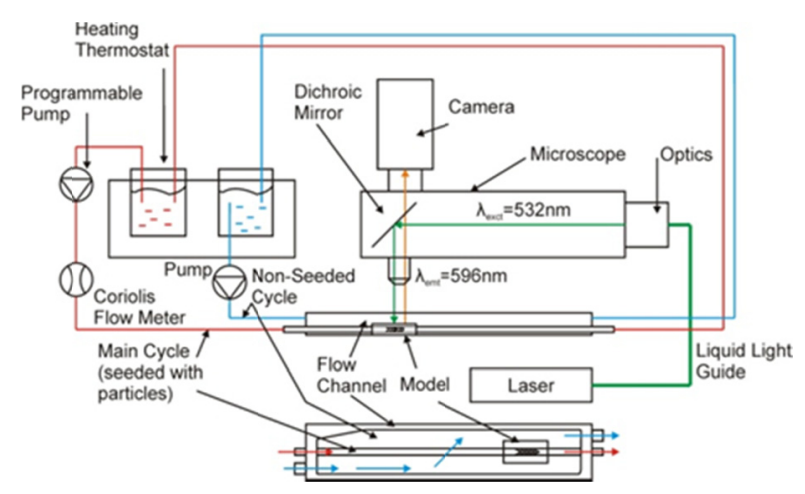

Figure 3: Experimental setup

\subsection{Flow Simulation}

For the numerical flow simulation the equation of momentum and mass conservation are solved. The shear thinning property of the fluid is considered by the Carreau-Yasuda model.

$$
\eta(\dot{\gamma})=\eta_{\infty}+\left(\eta_{0}-\eta_{\infty}\right)\left[1+(\lambda \dot{\gamma})^{a}\right]^{(n-1) / a}
$$

Here $\eta_{\infty}$ and $\eta_{0}$ are the minimum and maximum viscosity thresholds. The time constant $\lambda$ as well as the model parameters $a$ and $n$ describe the shear thinning behavior in between. The parameter values used for the numerical simulations are: $\eta_{\infty}=1.8 \cdot 10^{-6} \mathrm{~m}^{2} / \mathrm{s}, \eta_{0}=1.6 \cdot 10^{-5} \mathrm{~m}^{2} / \mathrm{s}$, $\lambda=0.606 s, a=0.874$ and $n=0.486$, regarding to the rheological properties of the analogue fluid, which have been measured previously.

The domain is discretized using the Finite Volume Method. To close the system of differential equations the following boundary conditions are defined. For the inlet condition a fully developed velocity is implemented as Dirichlet condition. The pressure gradient is set to zero at the inlet. The vessel wall and the stent struts are defined as noslip walls. Regarding to the symmetrical stent geometry only one sixth of the cross section has to be modeled. The corresponding surfaces are considered as symmetry planes. For pre- and post-processing as well as solving the open source software package OpenFOAM is used.

\section{Results}

\subsection{Structural Mechanics}

The results of the finite element analysis show that the investigated stents lead to a considerable stiffening of the 
stented vessel region. This compliance mismatch does not depend very much on the stent design. The main fraction of the compliance reduction is due to the stenosis; however, a detailed analysis shows that the local compliance is particularly reduced in the vicinity of the stent struts. Moreover, a tissue prolapse and an increased wall stress can be seen there (Fig. 4).

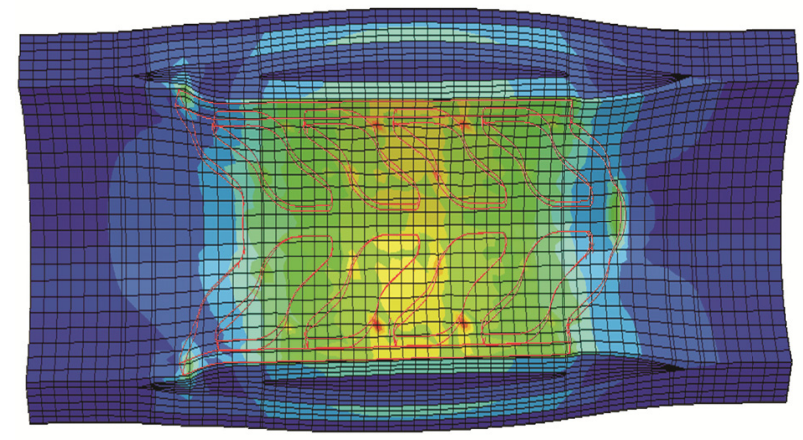

Figure 4: Detailed view of the stent showing increased v. Mises vessel wall stress

\subsection{Experimental fluid mechanics}

Because the wall shear stress is known to be the main determinant of the restenosis risk from a fluid mechanical point of view, wall shear stress distributions are discussed here. The experimental data are obtained in a single plane, so wall shear stress data can be gained for an intersecting line between measurement plane and vessel wall. Figure 5 illustrates the wall shear stress distribution of the three stent models considered.

For each model a strong decrease of the wall shear stress in the vicinity of the stent struts can be observed. Negative values indicate recirculation zones in front and between the struts. Up- and downstream the struts the wall shear stress reaches values close to the state of the undisturbed pipe flow.

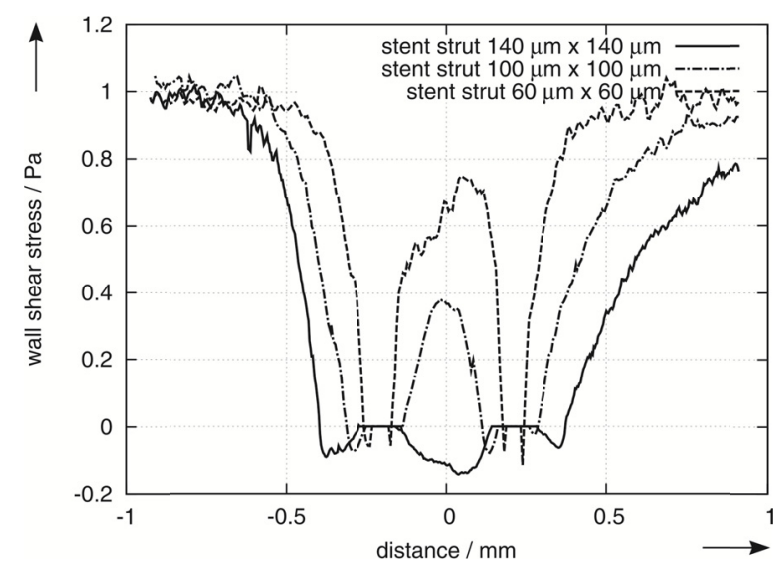

Figure 5: Experimental wall shear stress distribution

\subsection{Numerical fluid mechanics}

For a determination of the spatial wall shear stress distribution the numerical simulation is used. As a reference calculations for a non-Newtonian flow in a straight pipe were carried out. The obtained wall shear stress value is 1.1 Pa. Figure 6 shows the wall shear stress distribution on the vessel walls for the three model stents.

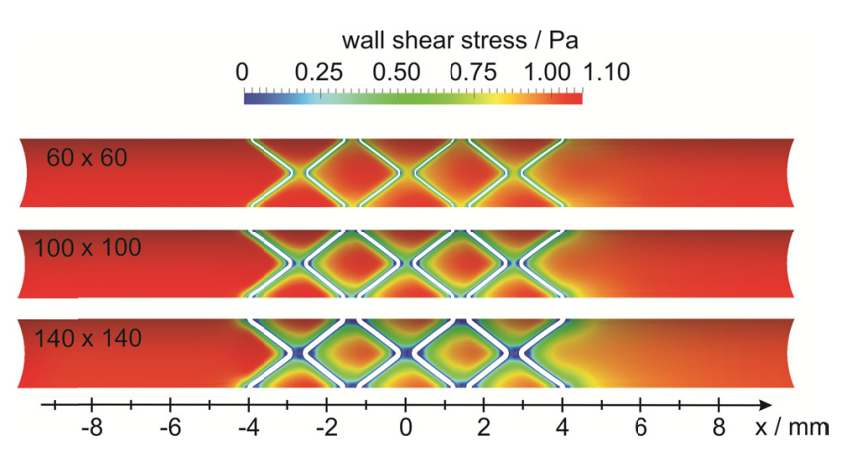

Figure 6: Wall shear stress distributions from numerical simulations of the flow in three model stents

From Figure 6 it can be clearly seen the wall shear stress upstream the first stent strut displays the same value as in a fully developed pipe flow. Between the stent struts the wall shear stress is below this value for all model stents. Nevertheless the stent design is a strong impact factor for wall shear stress distribution. The $60 \mu \mathrm{m} \times 60 \mu \mathrm{m}$ model stent clearly causes higher values in the stented vessel area than the other two model stents. For quantification the area covered by the stent strut as well as the area with wall shear stress below the critical value of $0.5 \mathrm{~Pa}$ is calculated and illustrated in Figure 7. The cross sections of the stent struts are approximately square, therefore the vessel area which is covered increases with increasing strut thickness. As a consequence of the increasing flow obstruction the recirculation zones and thereby' the areas of low wall shear stress become more prominent. The rate of increase of the wall shear stress area is higher than that of the covered vessel. Low cross section areas of the stent struts lead to a significant improvement regarding to in-stent the restenosis risk.

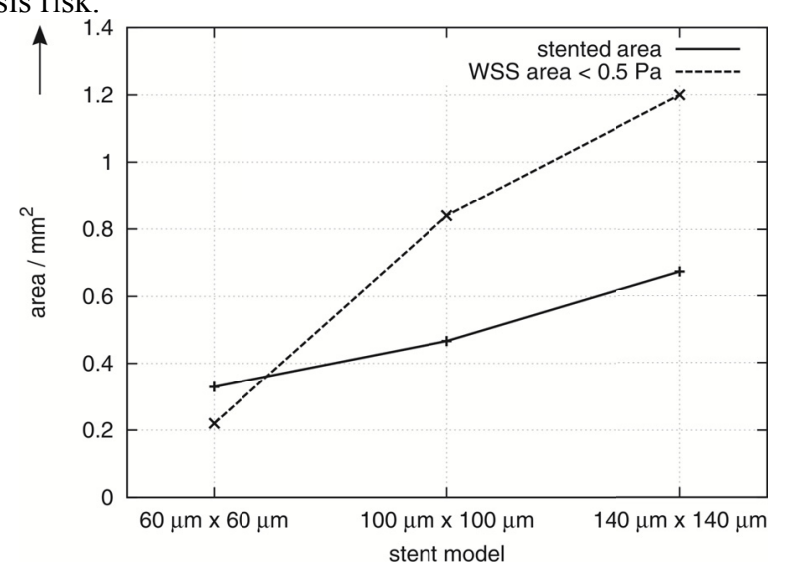

Figure 7: Area of low wall shear stress for three model stents 


\section{Conclusions}

The finite element investigations on the stent-stenosis system show that there is a considerable change of the stress and of the deformation in the vicinity of stents. Hence, the stent is causal for a change of structural parameters in the stented vessel region, such as compliance, deformation and stress. A degradable stent is therefore desirable, as it changes these parameters only temporarily. However, an optimization of all stents designs with respect to these parameters remains necessary. Finite element modeling studies are especially helpful with this task.

The fluid mechanical investigations show that there is a strong influence of the stent design on the wall shear stress distribution. Small struts with big distances in between should be preferred to allow a reattachement of the flow. This flow pattern then results in an increase of wall shear stress and a reduction of zones with low or negative wall shear stress and therefore benefits the patient.

\section{References}

[1] Balossina R., Gervaso F., Migiavacca F., Dubini G., Effects of different stent designs on local hemodynamics in stented arteries, Journal of Biomechanics 2008; 41: 1053-1061

[2] Berry J. L., Santamarina A., Moore Jr. J. E., Roychowdhury S., Routh W., Eperimental and Computational Flow Evaluation of Coronary Stents, Annals of Biomedical Engineering 2000; 28: 386398

[3] Brookshier K. A. and Tabell J. M., Evaluation of blood analog fluid:aqueous xanthan gum/glycerine, Biorheology 1993; 30: 107-116

[4] Budwig R., Refractive index matching methods for liquid flow investigations, Experiments in Fluids 1994, 17; 350-355

[5] Chien S., Shear dependence of effective cell volume as a determinant of blood viscosity, Science 1970; 168: $977-978$

[6] Gao Y. and Kilfoil M., Accurate detection and complete tracking of large populations of features in the three dimensions, Optics Express 2009; 17(6): 4685-4704

[7] Gibson C. M., Diaz L., Kandarpa K., Sacks F. M., Pasternak R. C., Sandor T., Feldman C., Stone P. H. Relation of Vessel Wall Shear Stress to Atherosclerosis Progression in Human Coronary Artries, Arteriosclerosis, Thrombosis and Vascular Biology, 1993; 13: 310-315

[8] Kastrati A., Mehilli J., Dirschinger J., Pache J., Ulm K., Schühlen H., Seyfarth M., Schmitt C., Blasini R., Neumann F.-J., Schömig A., Restenosis After Coronary Placement of Various Stent Types, The American Journal of Cardiology 2001; 87: 34-39

[9] Morlacchi S., Keller B., Arcangeli P., Balzan M., Migliavacca F., Dubini G., Gunn J., Arnold N.,
Narracott A., Evans D., Lawford P., Hemodynamics and In-stent Restenosis: Micro-CT Images, Histology and Computational Simulations, Annals of Biomedical Engineering 2001

[10] Nguyen T. T., Biadillah Y., Mongrain R., Brunette J., Tardif J. C. Bertrand O. F., A Method for Matching the Refractive Index and Kinematic Viscosity of a Blood Analog for Flow Visualisation in Hydraulic Cardiovascular Models, Journal of Biomechanical Engineering 2004; 126(4): 529-535

[11] Oh S, Kleinberger M, McElhaney JH. Finite-element analysis of balloonangioplasty, Med. \& Biol. Eng. \& Comput. 1994; 32 (Suppl. 1): 108-114. 